\title{
Article \\ Effect of Binding Modules Fused to Cutinase on the Enzymatic Synthesis of Polyesters
}

\author{
Valerio Ferrario ${ }^{1}\left({ }^{\infty}\right.$, Anamaria Todea ${ }^{1}\left(\mathbb{D}\right.$, Lisa Wolansky ${ }^{2}$, Nicola Piovesan ${ }^{1,2}$, Alice Guarneri ${ }^{1,2}$, \\ Doris Ribitsch 2,3 (1) Georg M. Guebitz ${ }^{2,3}[$, Lucia Gardossi $1, * \mathbb{(})$ and Alessandro Pellis $2,4, *$ (i)
}

1 Laboratory of Applied and Computational Biocatalysis, Department of Scienze Chimiche e Farmaceutiche, Università degli Studi di Trieste, Piazzale Europa 1, 34127 Trieste, Italy; valerio.ferrario@gmail.com (V.F.); atodea@units.it (A.T.); nicola.piovesan@yahoo.it (N.P.); alliguarneri@gmail.com (A.G.)

2 Institute of Environmental Biotechnology, Department of Agrobiotechnology IFA-Tulln, University of Natural Resources and Life Sciences, Konrad Lorenz Strasse 20, 3430 Tulln an der Donau, Austria; lisa.wolansky@gmail.com (L.W.); doris.ribitsch@boku.ac.at (D.R.); guebitz@boku.ac.at (G.M.G.)

3 Austrian Centre of Industrial Biotechnology, Division Enzymes \& Polymers, Konrad Lorenz Strasse 20, 3430 Tulln an der Donau, Austria

4 Dipartimento di Chimica e Chimica Industriale, Università degli Studi di Genova, Via Dodecaneso 31, 16146 Genova, Italy

* Correspondence: gardossi@units.it (L.G.); alessandro.pellis@unige.it or alessandro.pellis@boku.ac.at (A.P.)

\section{check for}

updates

Citation: Ferrario, V.; Todea, A.;

Wolansky, L.; Piovesan, N.; Guarneri,

A.; Ribitsch, D.; Guebitz, G.M.;

Gardossi, L.; Pellis, A. Effect of

Binding Modules Fused to Cutinase

on the Enzymatic Synthesis of

Polyesters. Catalysts 2022, 12, 303

https://doi.org/10.3390/

catal12030303

Academic Editors: Agnieszka

Siewniak and Anna Chrobok

Received: 4 February 2022

Accepted: 5 March 2022

Published: 7 March 2022

Publisher's Note: MDPI stays neutral with regard to jurisdictional claims in published maps and institutional affiliations.

Copyright: (C) 2022 by the authors. Licensee MDPI, Basel, Switzerland. This article is an open access article distributed under the terms and conditions of the Creative Commons Attribution (CC BY) license (https:// creativecommons.org/licenses/by/ $4.0 /)$.

\begin{abstract}
In relation to the development of environmentally-friendly processing technologies for the continuously growing market of plastics, enzymes play an important role as green and sustainable biocatalysts. The present study reports the use of heterogeneous immobilized biocatalysts in solventfree systems for the synthesis of aliphatic oligoesters with $M_{W} s$ and monomer conversions up to $1500 \mathrm{Da}$ and $74 \%$, respectively. To improve the accessibility of hydrophilic and hydrophobic substrates to the surface of the biocatalyst and improve the reaction kinetic and the chain elongation, two different binding modules were fused on the surface of cutinase 1 from Thermobifida cellulosilytica. The fusion enzymes were successfully immobilized ( $>99 \%$ of bound protein) via covalent bonding onto epoxy-activated beads. To the best of our knowledge, this is the first example where fused enzymes are used to catalyze transesterification reactions for polymer synthesis purposes.
\end{abstract}

Keywords: immobilized cutinases; enzyme engineering; polyesters; solvent-less biocatalyzed; polycondensation; sustainable chemistry

\section{Introduction}

When compared with classical chemical catalysis, enzymes frequently prevail in terms of selectivity and impact on the environment [1]. Biocatalysts were evolved towards the needs of nature as versatile catalysts but, in most cases, they do not match the current needs of industrial biotechnology processes [2]. One of the current most growing fields of interest is, without a doubt, the application of biocatalysts for the processing of natural and synthetic polymers, in particular polyesters [3,4]. These environmentally friendly catalysts can work under mild conditions, enabling both the surface functionalization of the polymer and its decomposition to constitutive monomers, which could in turn be used for a subsequent re-polymerization closing the carbon cycle. For such purposes, one of the most investigated enzyme class is cutinases, serine-hydrolytic enzymes that show activity not only on their natural substrate cutin but also on man-made polyesters [3]. The surface functionalization of poly(lactic acid) (PLA) by means of Humicola insolens cutinase (HiC) was recently reported for improved cytocompatibility and the enzymatic coupling of carboxylic acids on the newly generated surface hydroxyl groups [5,6]. Additionally, the hydrolysis of aromatic-aliphatic polyesters such as poly(ethylene terephthalate) (PET) $[7,8]$ and the promising poly(ethylene furanoate) (PEF) [9] were reported using cutinases from 
various Thermobifida species. More recently, the synthetic capabilities of cutinases were also exploited: HiC was demonstrated to be active in the synthesis of linear polyesters and ring opening polymerization of several lactones $[10,11]$ while cutinase 1 from Thermobifida cellulosilytica (Thc_Cut1) was reported as an interesting biocatalyst for the polycondensation of aliphatic oligoesters under solvent-free conditions [12].

Despite the versatility of such biocatalysts both for hydrolytic and synthetic reactions, further efforts need to be made to elucidate and expand the substrate range and the associated enzyme performance. The aim is to expand the pool of suitable building blocks from renewable sources for biopolymer applications. One of the preferred approaches in this direction, together with the discovery of new biocatalysts, is enzyme engineering that can be performed via directed evolution, rational and/or de-novo design or hybrid strategies [2,13]. For the cutinases-specific case, rational strategies were implemented to improve catalytic activity, mainly for PET degradation purposes. Thermobifida fusca cutinase was engineered in several approaches in order to change the polarity of the enzyme surface, relieve product inhibition and improve thermostability [14-16]. Likewise, cutinase 2 from Thermobifida cellulosilytica was also engineered in terms of its electrostatic and hydrophobic surface properties in the proposed polymer binding site [17]. All these alterations of the surface properties were designed to improve the enzymatic hydrolysis of polyesters. Moreover, several works on cutinase fusion proteins were also found, describing how different binding modules can lead to an increase of the catalytic activity at the interface $[18,19]$ This last approach is of particular interest because it underlines the importance of understanding enzyme-substrate interactions. In fact, it was assumed that the stimulatory effect of hydrophobins on cutinase-catalyzed PET hydrolysis is due to the creation of a hydrophobic coating at the target surface [20].

While for polyesters hydrolysis (in particular PET), several efforts were done in the last years, from the synthetic point of view, to the best of our knowledge; the only data related to an engineered enzyme for polyester synthesis was reported by Takwa et al. in 2011. This work describes a rational redesign approach on Candida antarctica lipase B, the most known biocatalysts for polyesters synthesis [3,21], for the improved ring opening polymerization of D,D-lactide [22].

In this research article we propose the utilization of Thc_Cut1 fused with different binding modules-from cellobiohydrolase I from Hypocrea jecorina (CBM) and an Alcaligenes faecalis poly(hydroxy alkanoate) depolymerase (PBM) — for the tuning of biocatalyzed polycondensation of aliphatic polyesters. The selected engineered enzymes were previously reported by Ribitsch et al. for the hydrolysis of PET [18] but their potential for synthesis have not yet been exploited.

\section{Results}

\subsection{Covalent Immobilization of the Biocatalyst}

Thermobifida cellulosilytica cutinase 1 fused with the hydrophilic binding module from cellobiohydrolase I from Hypocrea jecorina (Thc_Cut1_CBM) and with the more hydrophobic binding module from an Alcaligenes faecalis poly (hydroxy alkanoate) depolymerase (Thc_Cut1_PBM) were immobilized on epoxy-activated polymethacrylate spherical beads.

Figure 1 shows that after $1 \mathrm{~h}, 80 \%$ of Thc_Cut1_CBM (Figure 1A) and 79\% of Thc_Cut1_PBM (Figure 1B) were bound to the carrier. After $24 \mathrm{~h}$ of reaction, both enzymes show a $>99 \%$ protein binding onto the polymeric carrier, as determined via supernatant activity (Figure 1A,B), and a 95\% and 97\% for Thc_Cut1_CBM and Thc_Cut1_PBM, respectively, concerning protein concentration analysis (Figure 1C,D and Table 1). 

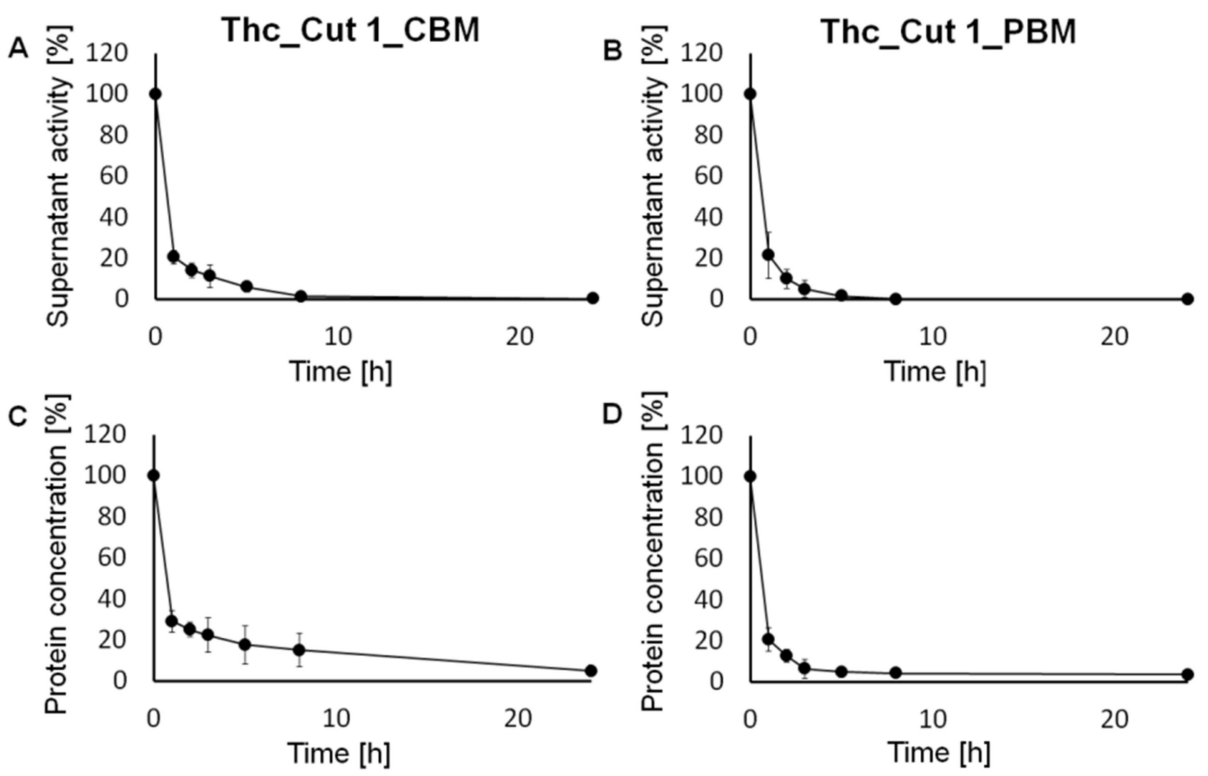

Figure 1. Thc_Cut1_CBM (A) and Thc_Cut1_PBM (B) immobilization profile on Resindion EC-EP/M epoxy-activated beads based on supernatant activity and Thc_Cut1_PBM (C) and Thc_Cut1_CBM (D) immobilization profile based on supernatant protein concentration. All experiments were performed in triplicates.

Table 1. Immobilization yields and recovered activities of Thc_Cut1_CBM and Thc_Cut1_PBM immobilized on EC-EP epoxy-activated beads using $10 \mathrm{mg}$ of protein per $\mathrm{g}$ of dry resin in $40 \mathrm{~mL}$ final volume. Immobilization was performed in $0.1 \mathrm{M}$ TRIS- $\mathrm{HCl}$ buffer $\mathrm{pH} 7$ at $21^{\circ} \mathrm{C}$ for $24 \mathrm{~h}$.

\begin{tabular}{cccc}
\hline Enzyme & \multicolumn{2}{c}{ Bound Enzyme (\%) } & Ref. \\
\hline Thc_Cut1 & $>99 *$ & $>99^{\delta}$ & {$[12]$} \\
\hline Thc_Cut1_PBM & $>99 *$ & $97^{\delta}$ & This work \\
Thc_Cut1_CBM & $>99 *$ & $95^{\delta}$ &
\end{tabular}

* Calculated by evaluating the residual activity in the supernatant via PNPB assay. ${ }^{\delta}$ Calculated by evaluating the residual protein concentration in the supernatant.

This immobilization behaviour, displaying $>99 \%$ of immobilized biocatalyst after only $5 \mathrm{~h}$ of reaction, resembles closely what was previously reported for the recombinant Thc_Cut1 when immobilized on the same support at the same pH using the same buffer [12] A possible rational explanation of this similarity is because the binding modules that were attached to the recombinant Thc_Cut1 are located at a certain distance from the lysine (Lys) residues located on the enzyme surface, which are most likely involved in covalent immobilisation. Moreover, the fused binding modules do not possess such lysine residues in their sequence (Figure 2). As previously reported, the primary amino groups of the superficial Lys are the main candidates for the formation of covalent bonds via the nucleophilic attack of the epoxy functionalities exposed on the polymeric support $[23,24]$. The superficial Lys residues are located far from the active site of Thc_Cut1, and this feature is expected to favour the correct orientation of the enzyme upon covalent binding as well as the active site accessibility to the substrates [12]. The two preparations will hence be referred to as Thc_Cut1_CBM and Thc_Cut1_PBM, respectively. 

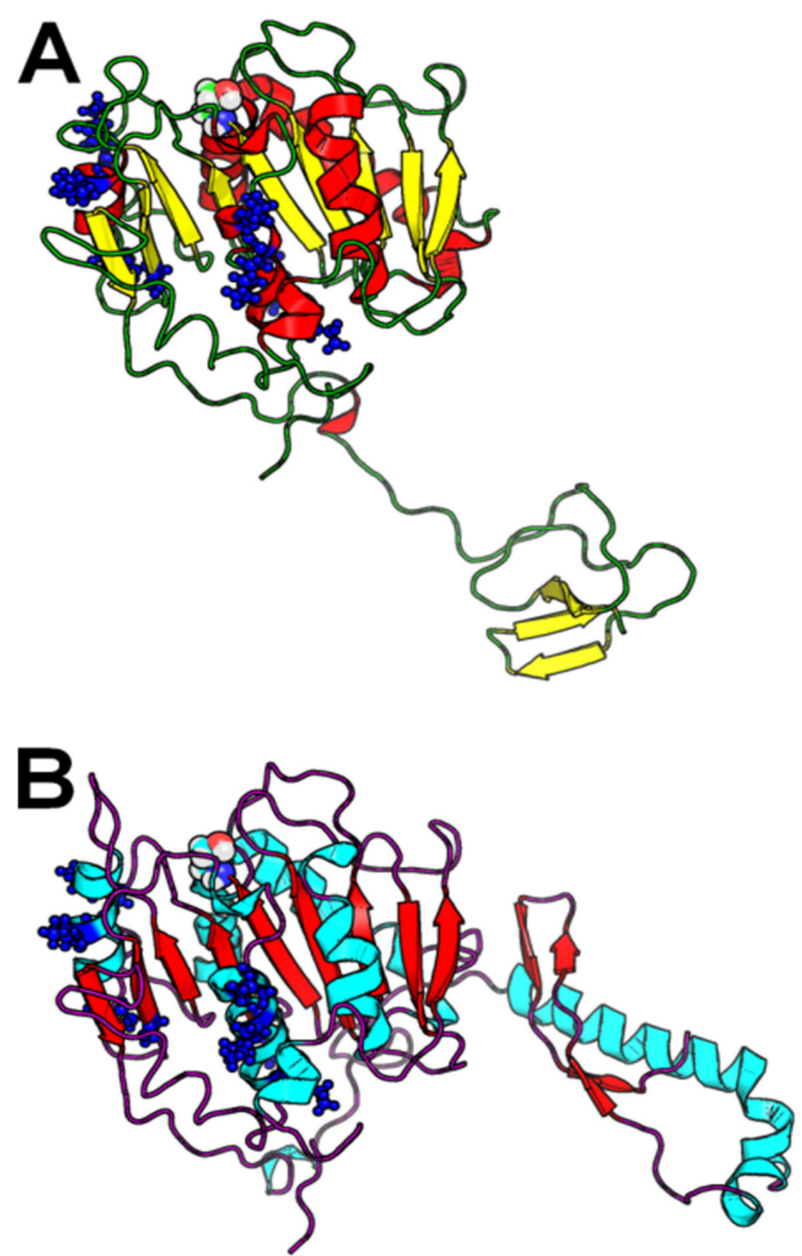

Figure 2. Lysine residues on the Thc_Cut1_CBM (A) and the Thc_Cut1_PBM (B) surface, highlighted in blue sphere mode. The catalytic serine residue (Ser131) was also highlighted in sphere mode.

\subsection{Polycondensation Reactions in Bulk Systems}

After the covalent immobilization of the biocatalyst, in a next step, enzymatic polycondensation was investigated. Previous reports on the synthetic characteristics of Thc_Cut1 and thin-film solvent-free reaction systems were taken as starting points for this study [25].

Reactions conducted at the same conditions previously reported for Thc_Cut1 [12] (70 ${ }^{\circ} \mathrm{C}$ and 1000 mbar) confirmed that also the cutinases fused to binding modules, when used in bulk systems at the previously mentioned conditions, were way more effective in converting the $C 4$ linear 1,4-butanediol (BDO) when compared with the C8 diol 1,8octanediol (ODO) (Table 2). In fact, Thc_Cut1_CBM and on the Thc_Cut1_PBM lead to conversion rates of $56 \%$ and $21 \%$ for BDO and ODO, respectively (Table 2 and Figure 3A,B).

Table 2. Polycondensation of DMA with various diols by different covalently-immobilized fusion hydrolases at $24 \mathrm{~h}$ at $70{ }^{\circ} \mathrm{C}$ and $1000 \mathrm{mbar}$, using $10 \% \mathrm{w} \mathrm{w}^{-1}$ of biocatalyst.

\begin{tabular}{cccccc}
\hline Preparation & Diol & Conversion $[\%]^{\kappa}$ & $\mathbf{M}_{\mathbf{w}}{ }^{*}$ & $\mathbf{M}_{\mathbf{n}}{ }^{*}$ & PD $^{*}$ \\
\hline \multirow{2}{*}{ iCBM } & BDO & 56 & 300 & 200 & 1.44 \\
& ODO & 21 & 300 & 300 & 1.13 \\
\multirow{2}{*}{ iPBM } & BDO & 56 & 500 & 500 & 1.11 \\
& ODO & 21 & 400 & 300 & 1.20 \\
\hline
\end{tabular}

${ }^{K}$ Calculated via ${ }^{1} \mathrm{H}-\mathrm{NMR}$ by comparing the ratio between the diol methylene groups adjacent to the -OH area and the internal methylene groups' area of DMA (assumed as constant). * Calculated via GPC calibrated with low molecular weight polystyrene standards ranging from 250-70,000 Da. All reactions were performed in duplicates. 

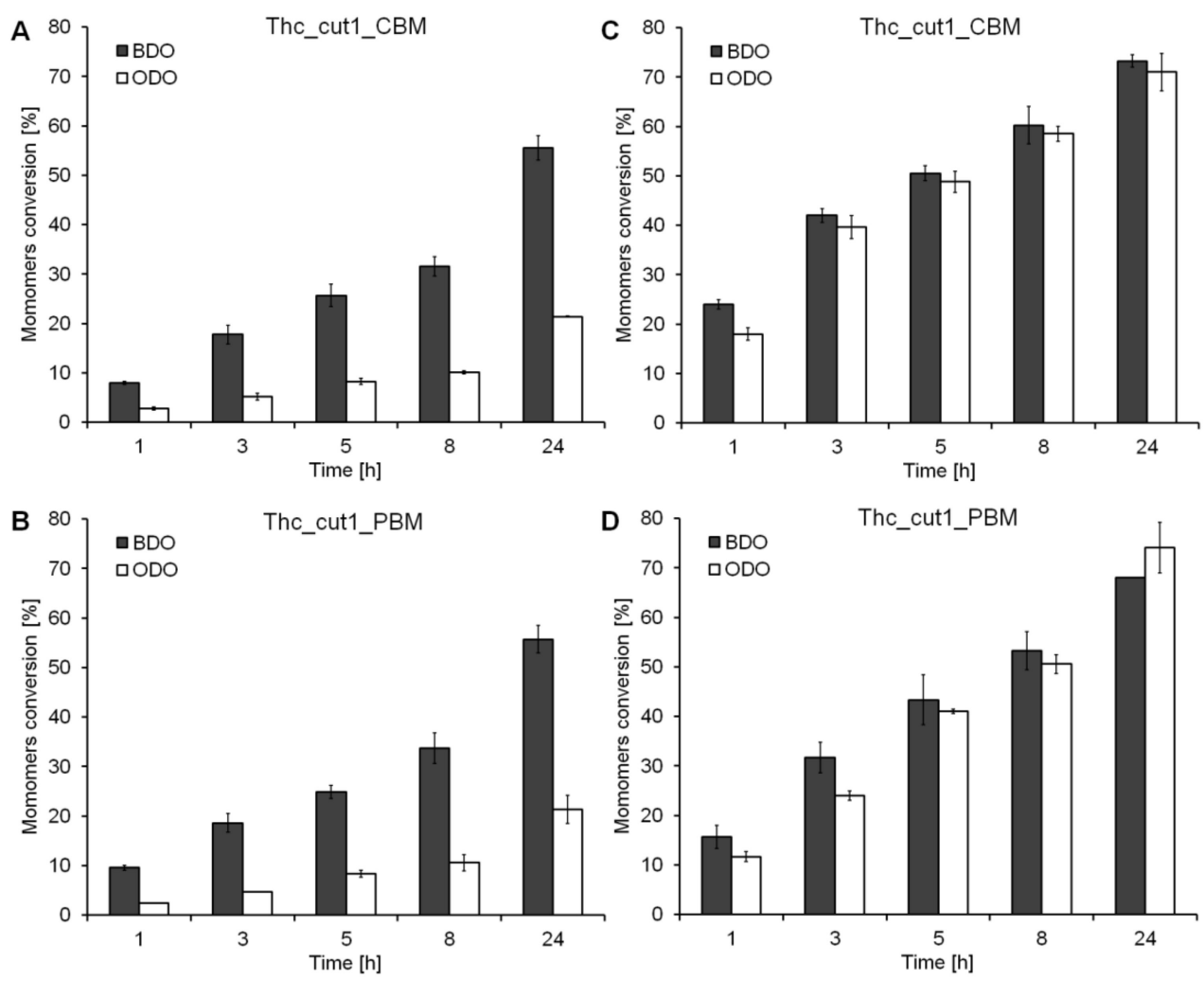

Figure 3. Enzymatic polycondensations of DMA and BDO or ODO catalyzed at $70{ }^{\circ} \mathrm{C}(\mathbf{A}, \mathbf{B})$ or $50{ }^{\circ} \mathrm{C}$ (C,D) using two different cutinases fused with binding domains (Thc_Cut1_CBM, Thc_Cut1_PBM).

This preference for the $\mathrm{C} 4$ vs the $\mathrm{C} 8$ linear diol when reacting at $70{ }^{\circ} \mathrm{C}$ was already reported for the recombinant Thc_Cut1 in the polycondensation reaction with DMA [12].

When the reaction temperature was instead lowered to $50{ }^{\circ} \mathrm{C}$, monomer conversions around $70 \%$ (Figure 3C,D) and molecular weights up to $\sim 1500$ Da were achieved with both Thc_Cut1_CBM and Thc_Cut1_PBM (Table 3), a value that is very similar to the 1900 Da obtained with the recombinant Thc_Cut1 described in a previous work.

Table 3. Polycondensation of DMA with various diols by different covalently-immobilized cutinases fused with binding modules at $24 \mathrm{~h}$ at $50{ }^{\circ} \mathrm{C}$ and $1000 \mathrm{mbar}$, using $10 \% \mathrm{w} \mathrm{w}^{-1}$ of biocatalyst.

\begin{tabular}{lccccc}
\hline Preparation & Diol & Conversion [\%] ${ }^{\kappa}$ & $\mathbf{M}_{\mathbf{w}}{ }^{*}$ & $\mathbf{M}_{\mathbf{n}}{ }^{*}$ & $\mathbf{D}^{*}$ \\
\hline \multirow{2}{*}{ Thc_Cut1_CBM } & BDO & 67 & 700 & 600 & 1.29 \\
& ODO & 60 & 1500 & 1000 & 1.59 \\
\hline \multirow{2}{*}{ Thc_Cut1_PBM } & BDO & 68 & 900 & 700 & 1.37 \\
& ODO & 74 & 1600 & 1000 & 1.53 \\
\hline
\end{tabular}

${ }^{{ }^{K} \text { Calculated via }}{ }^{1} \mathrm{H}$-NMR by comparing the ratio between the diol methylene groups adjacent to the -OH area and the internal methylene groups' area of DMA (assumed as constant). * Calculated via GPC calibrated with low molecular weight polystyrene standards ranging from 250-70,000 $\mathrm{Da}$. All reactions were performed in duplicates.

Both reaction mixtures, DMA-BDO and DMA-ODO lead to viscous solutions at the chosen operational temperature. Being a polycondensation reaction with the formation of macromolecules, the viscosity of the systems is constantly changing with the greatest influence on the enzymatic polymerization that is therefore given by the temperature and not from the fused binding modules that have a negligible effect on the polycondensation progression. 


\subsection{Enzyme Modelling \& Surface Analysis}

The two enzyme structures: Thc_Cut1_CBM and Thc_Cut1_PBM were obtained by Rosetta Ab initio calculation [26]. A second set of enzyme structures was calculated by a full de novo structure prediction using the Robetta server [27] to have insights into the reliability of the ab initio models. By structural superimposition, it is evident that, while the core of the Thc_Cut1 protein is in good agreement in all the modelled structures (the RMDS values related to the core enzyme structure were lower than 0.55 in both cases), the fused binding modules are attached to Thc_Cut1 via a long coil region. Interestingly, for CBD, there seems to be some interface interactions between the two protein domains as predicted by Robetta (see ESI, Figures S1-S5), which can potentially lead to a less accessible binding site which is, on the other hand, something we do not notice from the experimental data since the activity of both enzyme chimeras is overall comparable. The ab initio structures were then used for the following Molecular Dynamics (MD) steps starting by a structure equilibration of $5 \mathrm{~ns}$, in an explicit aqueous environment using the TIP4p water model [28], followed by $5 \mathrm{MD}$ simulation runs for a total of $25 \mathrm{~ns}$ at $300 \mathrm{~K}$ using the GROMACS software version 4 [29]. At the end of each $5 \mathrm{~ns}$ MD run, the input for the subsequent MD ran was determined by performing a structural clustering to identify the median structure of the most populated cluster. The outcome of the simulations highlights essential structural stability in the original Thc_Cut1 structure which is evident by comparing each enzyme structure in its initial situation with its final equilibrated structure (Figure 4).

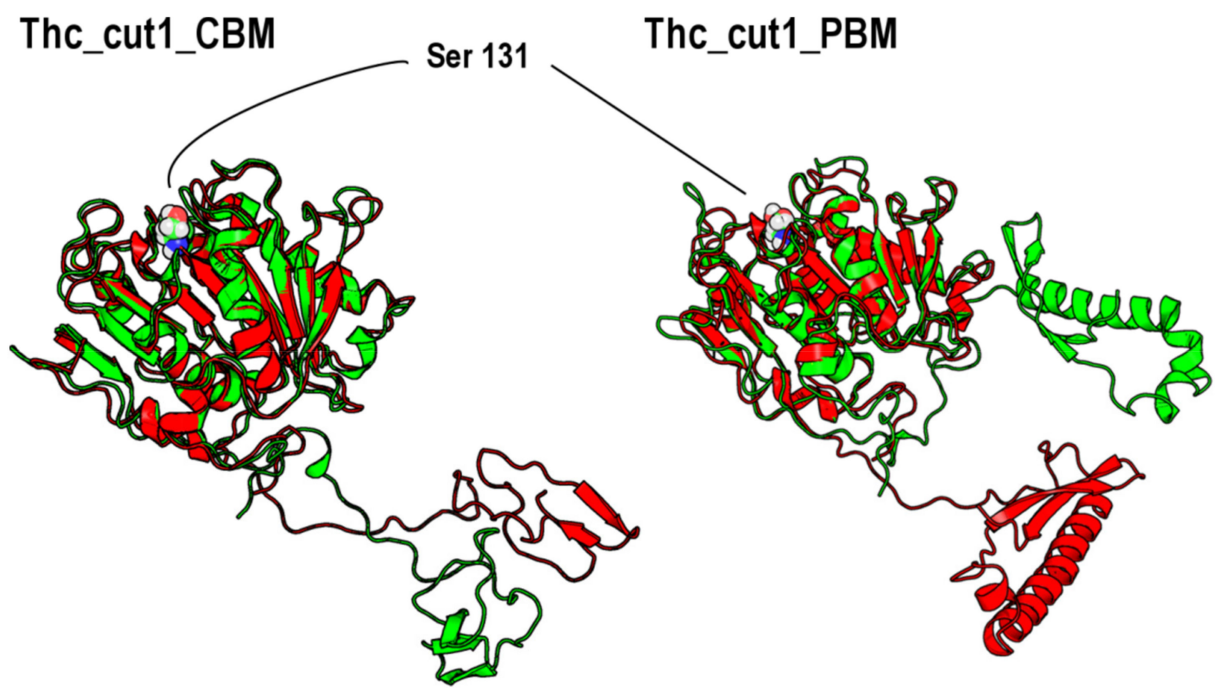

Figure 4. Initial (in red) and final (in green) structures of Thc_Cut1_CBM and Thc_Cut1_PBM after 25 ns of MD simulation. Protein structures are represented in cartoon mode; the catalytic serine is represented in sphere mode and labelled.

The original Thc_Cut1 core was stable during the simulation, whereas structural changes are evident for the binding domains, especially related to the "random coil" link region, even if the general secondary structure organization was retained, in agreement with the previous findings from the different homology modelling strategies.

The final equilibrated structures coming from MD simulation were subjected to surface analysis using the GRID method [30] (Figure 5). 


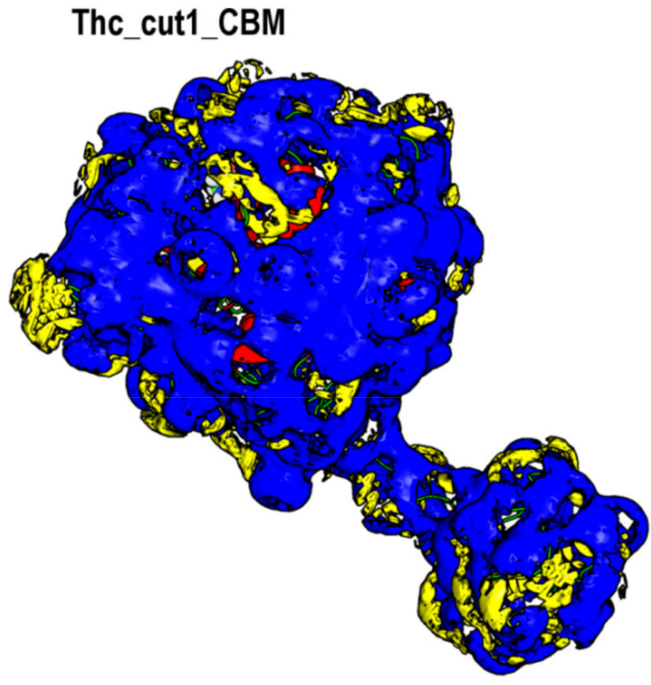

Hydrophobic surface

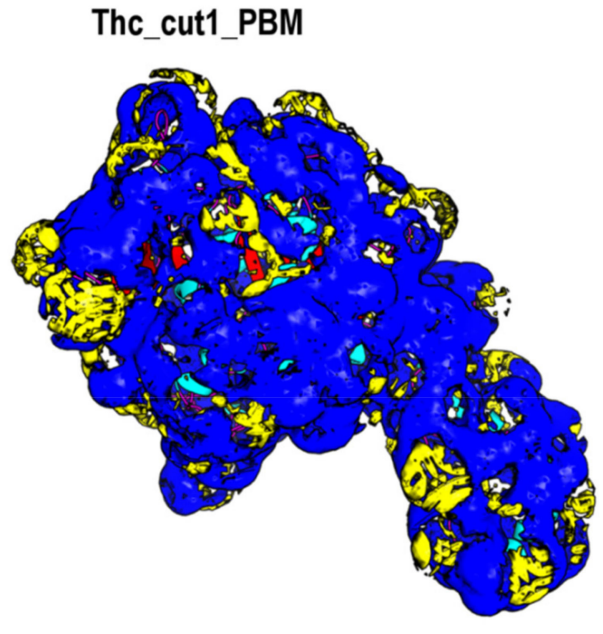

Hydrophilic surface

Figure 5. Surface analysis of Thc_Cut1_CBM and Thc_Cut1_PBM after MD equilibration. Hydrophobic surfaces are highlighted in yellow, hydrophilic surfaces are indicated in blue.

The superficial properties of the two enzyme structures appear to be similar to each other, and the analysis of Figure 5 shows that the two structures have mainly a hydrophilic nature. A possible difference in terms of hydrophobicity of the two binding domains was previously hypothesized by Ribitsch et al. [18] to explain the different performances of the two different chimeric enzymes in the PET hydrolysis, since the two different binding modules were developed by Nature to bind cellulose (CBD) and PHB (PBM), respectively. Nevertheless, the analysed surfaces are indeed very similar in terms of hydrophilicity, therefore it is not possible to identify the hydrophobic/hydrophilic balance as responsible for different enzyme performances which are indeed very similar in polycondensation reactions, as reported in this paper.

The negligible differences in terms of hydrophilic and hydrophobic properties of the modules are in line with the similar behaviour and specificity observed for the two fused cutinases.

\subsection{Docking Analysis}

To get more details about the Thc_Cut1 variants substrates specificity, two dimers, BDO-DMA and ODO-DMA were docked to the catalytic site of the variants. To be precise, it is not possible to exclude any long-rearrange effect due to the fusion extra-domain, since there might be differences that can appear rather small but that can potentially lead to big differences in terms of substrate accommodation. Without the crystal structures of the fusion proteins, it is unfortunately not possible to exclude this hypothesis. The docking analysis was performed by using an empirical free-energy force field and rapid Lamarckian genetic algorithm search methods implemented in Autodock [31]. All the resulting poses corresponding to the lowest docking free energies were analysed, and the results are included in Table 4 . Results suggest that the shorter ligands (BDO-DMA) are better accommodated in the active sites of both variants, compared to ODO-DMA and even though the docking free energies values obtained for both variants are comparable, a higher clustering number was obtained in the case of Thc_Cut1_PBM. 
Table 4. AutoDock data relative to the docking free energies computed only for the poses corresponding to the lowest energy values.

\begin{tabular}{llcc}
\hline Mutant & Substrate & E* $_{\text {abs }}^{*}[\mathbf{k c a l} / \mathbf{m o l}]$ & Clustering \\
\hline Thc_Cut1_PBM & BDO-DMA & -3.34 & 53 \\
& ODO-DMA & -4.04 & 29 \\
Thc_Cut1_CBM & BDO-DMA & -3.36 & 12 \\
& ODO-DMA & -2.22 & 39 \\
\hline
\end{tabular}

For ODO-DMA substrate the docking free energies were negatively higher (about two times) and for this reason, the values can be associated with more favourable conformations. Overall, the results obtained for Thc_Cut1_PBM are slightly better when compared to Thc_Cut1_CBM and are in concordance with the experimental data presented in Table 3.

An explanatory example of the poses corresponding to the lowest docking energies for the BDO-DMA and ODO-DMA substrates in the catalytic sites of Thc_Cut1_PBM and Thc_Cut1_CBM variants are reported in Figure 6. It can be clearly observed that both substrates assume a favourable conformation in both variants; however, when compared to ODO-DMA, for BDO-DMA the orientation of the substrate in the catalytic site appears to be more favourable. This favourable orientation could be since BDO has less conformational possibilities, resulting in a more restricted set of possible poses and therefore of "productive" interactions in the enzyme's active site.

(a)

(c)

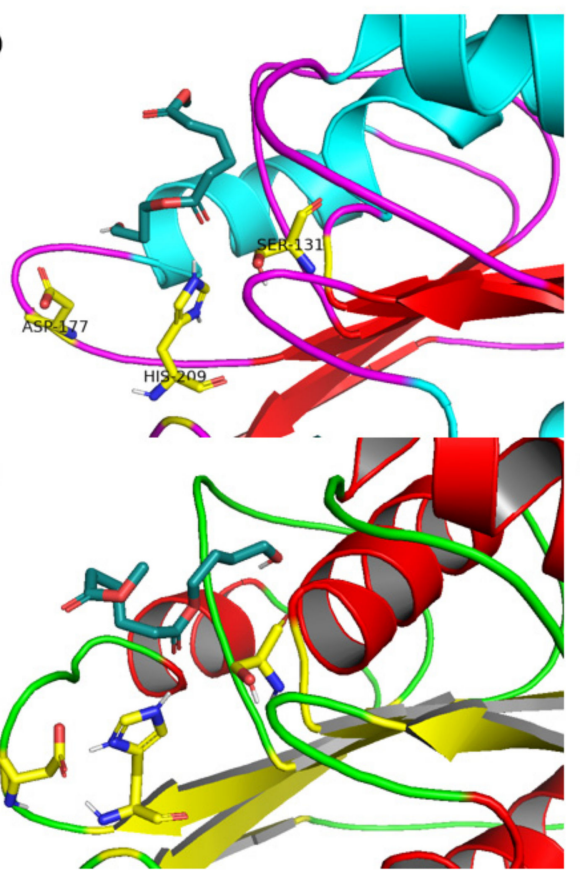

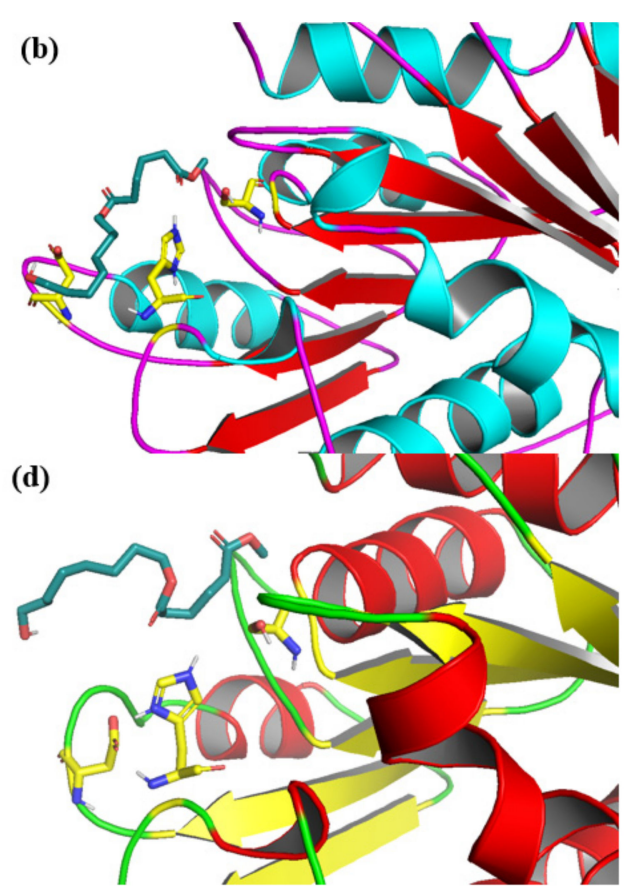

Figure 6. BDO-DMA $(\mathbf{a}, \mathbf{c})$ and ODO-DMA $(\mathbf{b}, \mathbf{d})$ substrates docked by Autodock in the active site of Thc_Cut1_PBM (a,b) and Thc_Cut1_CBM (c,d). The catalytic Ser 131, Asp 177 and His 209 are in yellow, whereas the substrates are green.

\section{Materials and Methods}

\subsection{Chemicals and Reagents}

EC-EP/M Sepabeads were purchased from Resindion S.r.l., (Mitsubishi Chemical Corporation, Milan, Italy), characterized by an average pore diameter of $10-20 \mathrm{~nm}$, particle size of $200-500 \mu \mathrm{m}$ and water retention of $55-65 \%$. Dimethyl adipate (DMA, $\geq 99 \%$ ), 1,4butanediol (BDO, ReagentPlus ${ }^{\circledR}, 99 \%$ ) and 1,8-octanediol (ODO, 98\%) were purchased from Sigma-Aldrich/Merck. All other chemicals and solvents were also purchased from 
Sigma-Aldrich at reagent grade and used without further purification if not otherwise specified.

\subsection{Enzymes}

The recombinant Thermobifida cellulosilytica cutinase 1 (Thc_Cut1) fused with the binding module from cellobiohydrolase I from Hypocrea jecorina (CBM) or with the binding module from Alcaligenes faecalis poly (hydroxy alkanoate) depolymerase (PBM) were produced and purified as previously described $[7,18]$. The organism used for the expression of the fusion enzymes was E. coli.

\subsection{Activity Assay for Free and Immobilized Cutinases}

The hydrolytic activity was measured at $21^{\circ} \mathrm{C}$ using $p$-nitrophenyl butyrate (PNPB) as substrate, as previously reported by Pellis et al. [5]. The activity was calculated in units $(\mathrm{U})$, where one unit is defined as the amount of enzyme required to hydrolyze $1 \mu \mathrm{mol}$ of substrate per minute under the given assay conditions using a 96-well plate reader (Infinite 200 Pro; Tecan, Männedorf, Switzerland).

\subsection{Protein Quantification}

Protein concentrations were determined as previously described [5] using an Infinite 200 Pro Tecan plate reader. The BioRad protein assay (Bio-Rad Laboratories GmbH, Vienna, Cat.No: 500-0006) was used taking bovine serum albumin as standard. The absorption after $5 \mathrm{~min}$ was measured at $\lambda=595 \mathrm{~nm}$ and the concentrations were calculated from the average of triplicate samples and blanks.

\subsection{Immobilization of Thc_Cut1_CBM and Thc_Cut1_PBM on Epoxy-Activated Beads}

The immobilization procedure was performed as previously reported [21]. The immobilization progress was monitored by evaluating the residual activity and protein concentration in the supernatant. After immobilization, the enzyme preparations were washed three times with $5 \mathrm{~mL}$ of $0.1 \mathrm{M}$ Tris- $\mathrm{HCl}$ buffer $\mathrm{pH} 7.0$ to remove all the non-covalently bound protein adsorbed on the support and dried for $48 \mathrm{~h}$ at $21^{\circ} \mathrm{C}$ under reduced pressure (100 $\mathrm{mm} \mathrm{Hg}$ ) in a desiccator containing silica gel prior to use. The immobilized preparations were named Thc_Cut1_PBM for the immobilized Thc_Cut1 fused with the PBM and Thc_Cut1_CBM for the immobilized Thc_Cut1 fused with the CBM.

\subsection{Enzymatic Polycondensation of DMA and Various Diols}

6.0 mmol DMA, 6.0 mmol of polyol (1,4-butanediol (BDO), 1,8-octanediol (ODO)), and the biocatalysts ( $10 \% \mathrm{w} \mathrm{w}^{-1}$ in respect to the total amount of monomers) were incubated in a 50-millilitre round bottom flask connected to a rotary evaporator at 50 or $70{ }^{\circ} \mathrm{C}$ and $1000 \mathrm{mbar}$ for $24 \mathrm{~h}$. The monomer molar ratio was 1.0:1.0. Before starting the polymerization process between DMA and ODO by adding the biocatalyst, the biphasic system was heated up to obtain a monophasic homogeneous transparent solution. The final product was solubilized in THF, and the immobilized enzyme was removed by filtration. After solvent evaporation, the crude product was analysed without further purification. All reactions were performed in duplicates. Control reactions in absence of the biocatalyst were also performed.

\subsection{GPC}

Samples were dissolved in THF and filtered through $0.2 \mu \mathrm{m}$ PFTE filters. Gel Permeation Chromatography (GPC) was carried out at $30{ }^{\circ} \mathrm{C}$ on an Agilent Technologies HPLC System (Agilent Technologies 1260 Infinity, Vienna, Austria) connected to a 17,369 $6.0 \mathrm{~mm}$ ID $\times 40 \mathrm{~mm}$ L HHR-H, $5 \mu \mathrm{m}$ Guard column and an 18,055 $7.8 \mathrm{~mm}$ ID $\times 300 \mathrm{~mm} \mathrm{~L}$ GMHHR-N, $5 \mu \mathrm{m}$ TSKgel liquid chromatography column (Tosoh Bioscience, Tessenderlo, Belgium) using THF (250 ppm BHT as inhibitor) as eluent (at a flow rate of $1 \mathrm{~mL} \mathrm{~min}^{-1}$ ). An Agilent Technologies G1362A refractive index detector was employed for detection. 
The molecular weights of the polymers were calculated using linear polystyrene calibration standards (250-70,000 Da).

\section{8. ${ }^{1} H-N M R$}

${ }^{1} \mathrm{H}$ Nuclear Magnetic Resonance (NMR) measurements were performed on a Bruker Avance II 400 spectrometer (resonance frequencies $400.13 \mathrm{MHz}$ for ${ }^{1} \mathrm{H}$ ) equipped with a 5-millimetre observe broadband probe head with z-gradients. $\mathrm{CDCl}_{3}$ was used as a solvent for all samples.

\subsection{Enzyme Structures}

Enzyme structures were obtained from Ribitsch et al. [18] that build them as follows: a model of Thc_Cut1 from Thermofibifida cellulosilytica DSM44535 was used; the model was previously generated in a different work obtained using Streptomyces exfoliatus lipase (PDB Code: 1JFR) as a template. As a suitable template for the PBM and CBD part is not available a template free modelling of PBM and CBD was performed using the molecular modelling suite Rosetta ab initio calculation as previously described using standard parameters [26]. The fasta sequences of both mutants are available in Supplementary Materials (Structure S1).

Moreover, to compare and evaluate the robustness of the obtained structures, a second set of models for the same protein sequences was obtained by submitting the sequences to the Robetta server, which performs a full de-novo structure prediction [27].

\subsection{Molecular Dynamic Simulations}

Each enzyme structure (Thc_Cut1_CBM and Thc_Cut1_PBM) was equilibrated by Molecular Dynamic (MD) simulation. The protonation state of each structure was calculated at $\mathrm{pH} 7.0$ by using the PDB2PQR server [32] based on the software PROPKA [33]. MD simulations were performed by using GROMACS software version 4 [29]. Each enzyme was defined into OPLS-AA force field [34] and placed into the centre of a cubic space of $343 \mathrm{~nm}^{3}$. Each system was solvated with explicit TIP4P water [35] and neutralized using $0.1 \mathrm{M} \mathrm{NaCl}$. Thus, each enzyme system was minimized using a steepest descendent algorithm for 10,000 steps. Iterative steps of MD simulation of $5 \mathrm{~ns}$ each were performed till a total simulation time of 25 ns. Every 5 ns of MD, g_cluster tool of the GROMACS package was used for the calculation of the most representative enzyme structure which was selected as starting point for the subsequent 5 ns MD step. MD simulations were all performed at $300 \mathrm{~K}$ in an NVT environment using the Particle Mesh Ewald (PME) algorithm [36] for the calculation of electrostatic interactions, v-rescale algorithm [37] for temperature and Berendsen algorithm for pressure [38].

\subsection{Surface Analysis}

Physical/Chemical properties in terms of surface hydrophilicity and hydrophobicity were calculated on the enzyme structures equilibrated by MD simulations. Surface analysis was performed using the software GRID [30] and visualized as isoenergy surfaces ( $-2.7 \mathrm{kcal} / \mathrm{mol}$ for hydrophobic interactions; $-0.7 \mathrm{kcal} / \mathrm{mol}$ for hydrophilic interactions).

\subsection{Docking Analysis}

The variants' structures obtained from the molecular dynamic studies were used for docking studies. Substrate molecules (dimers of dimethyl adipate and 1,4-butanediol and dimethyl adipate and 1,8-octanediol) were generated using ChemDraw and minimized using MOPAC [39] with the AM1 semiempirical method prior to docking. Auto-Dock version 4.2 [31] was used for performing the docking calculations with a Lamarckian genetic algorithm. GA runs were 100, GA population size was set at 1500 and a maximum number of evaluations at 25,000,000. For each experiment, a docking box $(60 \times 60 \times 60) \AA$, Grid Point Spacing $0.250 \AA$ centred on $\mathrm{t}$ using a Lamarckian genetic algorithm were used. The best candidates were visualized and further analysed using the software Pymol [40]. 


\section{Conclusions}

Two different binding modules were fused with the structure of cutinase 1 from Thermobifida cellulosilytica for tuning the efficiency of the enzyme in catalysing the polycondensation of polyesters with different hydrophilic and hydrophobic properties. The enzymes were successfully immobilized ( $>99 \%$ of bound protein) via covalent bonding on epoxy-activated carriers. Aliphatic oligoesters with $\mathrm{M}_{\mathrm{w}} \mathrm{s}$ up to $1500 \mathrm{Da}$ and monomer conversions up to $74 \%$ were obtained. The negligible differences in terms of hydrophilic and hydrophobic properties of the modules are in line with the similar behaviour and specificity observed for the two fused cutinases.

Docking studies shed light on the slight preference of the two cutinases for the shorter diol (BDO), although at a higher temperature, such a difference is negligible at the experimental level, suggesting different hypothesis: the physical-chemical properties of the reaction mixture-mainly viscosity-might play a major role in the reaction progression or the enzymes themself can access more accessible conformations leading to a decrease in chain length selectivity. Interestingly, the same chimeric enzymes showed a more pronounced difference in PET hydrolysis [18], indicating that the solvent environment may trigger different types of interactions.

Supplementary Materials: The following supporting information can be downloaded at: https: / / www.mdpi.com/article/10.3390/catal12030303/s1, Structure S1. Fasta files of Thc_Cut1 protein sequences; Table S1. RMSD values determined for each model resulted from Robetta server versus the initial protein structures and the structures resulted after MD; Figure S1. Superimposition of the CBM mutant structure before MD (green) and predicted model CBM1 resulted from Robetta server (turquoise); Figure S2. Superimposition of the CBD mutant structure before MD (green) and predicted model CBM 1 resulted from Robetta server (turquoise); Figure S3. Superimposition of the SBD mutant structure before MD (turquoise) and predicted SBM model 4 resulted from Robetta server (blue); Figure S4. Superimposition of the SBD mutant structure after MD (pink) and predicted model 4 resulted from Robetta server (blue); Figure S5. Superimposition of the SBD mutant structure after MD (pink) and predicted model 1 resulted from Robetta server (yellow).

Author Contributions: L.W. and N.P. expressed, purified, and immobilized the enzymes. L.W., N.P. and A.G. carried out the enzymatic synthesis. A.G., D.R., A.T. and V.F. carried out the bioinformatic analysis experiments. A.P., A.T. and V.F. wrote the manuscript. L.G., D.R. and G.M.G. revised the manuscript. L.G., G.M.G. and A.P. acquired the funding, supervised, and coordinated the project. All authors have read and agreed to the published version of the manuscript.

Funding: This project has received funding from the European Union's Horizon 2020 research and innovation program under the Marie Skłodowska-Curie grant agreement No. 101029444 and from the grant agreement No 953073, project UPLIFT (sUstainable PLastIcs for the Food and drink packaging indusTry).

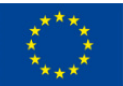

Data Availability Statement: All data are available in the ESI File or upon request from the corresponding authors.

Conflicts of Interest: The authors declare no conflict of interest.

\section{References}

1. De María, P.D. Biocatalysis, sustainability, and industrial applications: Show me the metrics. Curr. Opin. Green Sustain. Chem. 2021, 31, 100514. [CrossRef]

2. Steiner, K.; Schwab, H. Recent advances in rational approaches for enzyme engineering. Comput. Struct. Biotechnol. J. 2012, 2, e201209010. [CrossRef] [PubMed]

3. Pellis, A.; Acero, E.H.; Ferrario, V.; Ribitsch, D.; Guebitz, G.M.; Gardossi, L. The Closure of the Cycle: Enzymatic Synthesis and Functionalization of Bio-Based Polyesters. Trends Biotechnol. 2016, 34, 316-328. [CrossRef] [PubMed]

4. Sheldon, R.A.; Brady, D. Green Chemistry, Biocatalysis, and the Chemical Industry of the Future. ChemSusChem 2022, 202102628. [CrossRef] [PubMed] 
5. Pellis, A.; Acero, E.H.; Weber, H.; Obersriebnig, M.; Breinbauer, R.; Srebotnik, E.; Guebitz, G.M. Biocatalyzed approach for the surface functionalization of poly(L-lactic acid) films using hydrolytic enzymes. Biotechnol. J. 2015, 10, 1739-1749. [CrossRef]

6. Nyanhongo, G.S.; Rodríguez, R.D.; Prasetyo, E.N.; Caparrós, C.; Ribeiro, C.; Sencadas, V.; Lanceros-Mendez, S.; Acero, E.H.; Guebitz, G.M. Bioactive albumin functionalized polylactic acid membranes for improved biocompatibility. React. Funct. Polym. 2013, 73, 1399-1404. [CrossRef]

7. Acero, E.H.; Ribitsch, D.; Steinkellner, G.; Gruber, K.; Greimel, K.; Eiteljoerg, I.; Trotscha, E.; Wei, R.; Zimmermann, W.; Zinn, M.; et al. Enzymatic Surface Hydrolysis of PET: Effect of Structural Diversity on Kinetic Properties of Cutinases from Thermobifida. Macromolecules 2011, 44, 4632-4640. [CrossRef]

8. Barth, M.; Oeser, T.; Wei, R.; Then, J.; Schmidt, J.; Zimmermann, W. Effect of hydrolysis products on the enzymatic degradation of polyethylene terephthalate nanoparticles by a polyester hydrolase from Thermobifida fusca. Biochem. Eng. J. 2015, 93, 222-228. [CrossRef]

9. Pellis, A.; Haernvall, K.; Pichler, C.M.; Ghazaryan, G.; Breinbauer, R.; Guebitz, G.M. Enzymatic hydrolysis of poly(ethylene furanoate). J. Biotechnol. 2016, 235, 47-53. [CrossRef]

10. Feder, D.; Gross, R.A. Exploring Chain Length Selectivity in HIC-Catalyzed Polycondensation Reactions. Biomacromolecules 2010, 11, 690-697. [CrossRef]

11. Hunsen, M.; Abul, A.; Xie, W.; Gross, R. Humicola insolens cutinase-catalyzed lactone ring-opening polymerizations: Kinetic and mechanistic studies. Biomacromolecules 2008, 9, 518-522. [CrossRef] [PubMed]

12. Pellis, A.; Ferrario, V.; Zartl, B.; Brandauer, M.; Gamerith, C.; Acero, E.H.; Ebert, C.; Gardossi, L.; Guebitz, G.M. Enlarging the tools for efficient enzymatic polycondensation: Structural and catalytic features of cutinase 1 from: Thermobifida cellulosilytica Catal. Sci. Technol. 2016, 6, 3430-3442. [CrossRef]

13. Bloom, J.D.; Meyer, M.M.; Meinhold, P.; Otey, C.R.; MacMillan, D.; Arnold, F.H. Evolving strategies for enzyme engineering. Curr. Opin. Struct. Biol. 2005, 15, 447-452. [CrossRef] [PubMed]

14. Then, J.; Wei, R.; Oeser, T.; Barth, M.; Belisário-Ferrari, M.R.; Schmidt, J.; Zimmermann, W. Ca ${ }^{2+}$ and $\mathrm{Mg}^{2+}$ binding site engineering increases the degradation of polyethylene terephthalate films by polyester hydrolases from Thermobifida fusca. Biotechnol. J. 2015, 10, 592-598. [CrossRef] [PubMed]

15. Wei, R.; Oeser, T.; Schmidt, J.; Meier, R.; Barth, M.; Then, J.; Zimmermann, W. Engineered bacterial polyester hydrolases efficiently degrade polyethylene terephthalate due to relieved product inhibition. Biotechnol. Bioeng. 2016, 113, 1658-1665. [CrossRef]

16. Roth, C.; Wei, R.; Oeser, T.; Then, J.; Föllner, C.; Zimmermann, W.; Sträter, N. Structural and functional studies on a thermostable polyethylene terephthalate degrading hydrolase from Thermobifida fusca. Appl. Microbiol. Biotechnol. 2014, 98, 7815-7823. [CrossRef]

17. Herrero Acero, E.; Ribitsch, D.; Dellacher, A.; Zitzenbacher, S.; Marold, A.; Steinkellner, G.; Gruber, K.; Schwab, H.; Guebitz, G.M. Surface engineering of a cutinase from Thermobifida Cellulosilytica for improved polyester hydrolysis. Biotechnol. Bioeng. 2013, 110, 2581-2590. [CrossRef]

18. Ribitsch, D.; Yebra, A.O.; Zitzenbacher, S.; Wu, J.; Nowitsch, S.; Steinkellner, G.; Greimel, K.; Doliska, A.; Oberdorfer, G.; Gruber, C.C.; et al. Fusion of Binding Domains to Thermobifida cellulosilytica Cutinase to Tune Sorption Characteristics and Enhancing PET Hydrolysis. Biomacromolecules 2013, 14, 1769-1776. [CrossRef]

19. Zhang, Y.; Chen, S.; Xu, M.; Cavoco-Paulo, A.P.; Wu, J.; Chen, J. Characterization of thermobifida fusca cutinase-carbohydratebinding module fusion proteins and their potential application in bioscouring. Appl. Environ. Microbiol. 2010, 76, 6870-6876. [CrossRef]

20. Ribitsch, D.; Acero, E.H.; Przylucka, A.; Zitzenbacher, S.; Marold, A.; Gamerith, C.; Tscheließnig, R.; Jungbauer, A.; Rennhofer, H.; Lichtenegger, H.; et al. Enhanced cutinase-catalyzed hydrolysis of polyethylene terephthalate by covalent fusion to hydrophobins. Appl. Environ. Microbiol. 2015, 81, 3586-3592. [CrossRef]

21. Pellis, A.; Guarneri, A.; Brandauer, M.; Acero, E.H.; Peerlings, H.; Gardossi, L.; Guebitz, G.M. Exploring mild enzymatic sustainable routes for the synthesis of bio-degradable aromatic-aliphatic oligoesters. Biotechnol. J. 2016, 11, 642-647. [CrossRef] [PubMed]

22. Takwa, M.; Larsen, M.W.; Hult, K.; Martinelle, M. Rational redesign of Candida antarctica lipase B for the ring opening polymerization of d,d-lactide. Chem. Commun. 2011, 47, 7392-7394. [CrossRef] [PubMed]

23. Cantone, S.; Ferrario, V.; Corici, L.; Ebert, C.; Fattor, D.; Spizzo, P.; Gardossi, L. Efficient immobilisation of industrial biocatalysts: Criteria and constraints for the selection of organic polymeric carriers and immobilisation methods. Chem. Soc. Rev. 2013, 42, 6262-6276. [CrossRef] [PubMed]

24. Ferrario, V.; Ebert, C.; Knapic, L.; Fattor, D.; Basso, A.; Spizzo, P.; Gardossi, L. Conformational Changes of Lipases in Aqueous Media: A Comparative Computational Study and Experimental Implications. Adv. Synth. Catal. 2011, 353, 2466-2480. [CrossRef]

25. Pellis, A.; Corici, L.; Sinigoi, L.; D'Amelio, N.; Fattor, D.; Ferrario, V.; Ebert, C.; Gardossi, L. Towards feasible and scalable solventfree enzymatic polycondensations: Integrating robust biocatalysts with thin film reactions. Green Chem. 2015, 17, 1756-1766. [CrossRef]

26. Kaufmann, K.W.; Lemmon, G.H.; Deluca, S.L.; Sheehan, J.H.; Meiler, J. Practically Useful: What the Rosetta Protein Modeling Suite Can Do for You. Biochemistry 2010, 49, 2987-2998. [CrossRef]

27. Kim, D.E.; Chivian, D.; Baker, D. Protein structure prediction and analysis using the Robetta server. Nucleic Acids Res. 2004, 32, W526-W531. [CrossRef] 
28. Ferrario, V.; Pleiss, J. Simulation of protein diffusion: A sensitive probe of protein-solvent interactions. J. Biomol. Struct. Dyn. 2019, 37, 1534-1544. [CrossRef]

29. Hess, B.; Kutzner, C.; van der Spoel, D.; Lindahl, E. GROMACS 4: Algorithms for Highly Efficient, Load-Balanced, and Scalable Molecular Simulation. J. Chem. Theory Comput. 2008, 4, 435-447. [CrossRef]

30. Goodford, P.J. A Computational Procedure for Determining Energetically Favorable Binding Sites on Biologically Important Macromolecules. J. Med. Chem. 1985, 28, 849-857. [CrossRef]

31. Morris, G.M.; Ruth, H.; Lindstrom, W.; Sanner, M.F.; Belew, R.K.; Goodsell, D.S.; Olson, A.J. AutoDock4 and AutoDockTools4: Automated docking with selective receptor flexibility. J. Comput. Chem. 2009, 30, 2785-2791. [CrossRef] [PubMed]

32. Dolinsky, T.J.; Nielsen, J.E.; McCammon, J.A.; Baker, N.A. PDB2PQR: An automated pipeline for the setup of Poisson-Boltzmann electrostatics calculations. Nucleic Acids Res. 2004, 32, W665-W667. [CrossRef] [PubMed]

33. Søndergaard, C.R.; Olsson, M.H.M.; Rostkowski, M.; Jensen, J.H. Improved Treatment of Ligands and Coupling Effects in Empirical Calculation and Rationalization of pKa Values. J. Chem. Theory Comput. 2011, 7, 2284-2295. [CrossRef] [PubMed]

34. Kaminski, G.A.; Friesner, R.A.; Tirado-Rives, J.; Jorgensen, W.L. Evaluation and Reparametrization of the OPLS-AA Force Field for Proteins via Comparison with Accurate Quantum Chemical Calculations on Peptides. J. Phys. Chem. B 2001, 105, 6474-6487. [CrossRef]

35. Jorgensen, W.L.; Chandrasekhar, J.; Madura, J.D.; Impey, R.W.; Klein, M.L. Comparison of simple potential functions for simulating liquid water. J. Chem. Phys. 1983, 79, 926. [CrossRef]

36. Essmann, U.; Perera, L.; Berkowitz, M.L.; Darden, T.; Lee, H.; Pedersen, L.G. A smooth particle mesh Ewald method. J. Chem. Phys. 1995, 103, 8577. [CrossRef]

37. Bussi, G.; Donadio, D.; Parrinello, M. Canonical sampling through velocity rescaling. J. Chem. Phys. 2007, 126, 014101. [CrossRef]

38. Berendsen, H.J.C.; Postma, J.P.M.; van Gunsteren, W.F.; DiNola, A.; Haak, J.R. Molecular dynamics with coupling to an external bath. J. Chem. Phys. 1984, 81, 3684. [CrossRef]

39. Stewart, J.J.P. Optimization of parameters for semiempirical methods V: Modification of NDDO approximations and application to 70 elements. J. Mol. Model. 2007, 13, 1173-1213. [CrossRef]

40. DeLano, W.L. The PyMOL Molecular Graphics System; Delano Scientific, S.C., Ed.; 2002. Available online: https://pymol.org/2/ (accessed on 3 February 2022). 